\section{Lymphatic vessel system in gingival peri-implant tissues in humans}

Marchetti C, Poggi P, Reguzzoni M, Icaro Cornaglia A, Rodriguez Y Baena R: Lymphatic vessel system in gingival peri-implant tissues in humans. $J$ Periodont Res 1999; 34: 229-23I. Munksgaard 1999.

\section{Marchetti' P. Poggi ${ }^{2}$, \\ M. Reguzzoni', A. Icaro Cornaglia', Y. Rodriguez R. Baena ${ }^{3}$}

${ }^{1}$ Istituto di Istologia ed Embriologia Generale, 2Dipartimento di Scienze Morfologiche, Eidologiche e Cliniche, Sezione di Anatomia Umana, Istituto di Discipline Odontostoma tologiche Universita' di Pavia, Italia

Caria Marchetti, Istituto di Istologia ed

Embriologia Generale, via Forlanini 10, 27100

Pavia, Italy

Tet: 0382507272

Fax: 0382528330

e-mail: carta.marchetti@ unipv.it

Key words: human gingiva; peri-implant tissue; lymphatic vessels; ultrastructure

Accepted for publication March 16, 1999
Soft peri-implant tissue health is of primary importance in the success of implants supporting crowns and bridges. The abutment/fixture/soft tissue interface is one of the most critical points because, owing to the lack of an epithelial attachment, there is no barrier to microbial penetration towards the bone. It follows that possible marginal inflammation of the gingiva (peri-implantitis) can spread towards the apex causing bone resorption. The destruction and remodelling of the mucosal tissues following implant surgery produce extracellular matrix degradation (1). Therefore, an efficient system, able to remove the products of inflammatory or regenerative processes is essential to peri-implant soft tissue functionality. The lymphatic system plays a fundamental role in maintaining tissue integrity by removing excess fluids, macromolecules and interstitial material from the tissues. Nevertheless, to date few studies have been performed on the characteristics of lymphatic drainage in the periodontal tissues (2-4) and no studies have been conducted on the existence and characteristics of lymphatic drainage in the restored peri-implant mucosa. The aim of the present study was to investigate the existence, distribution and morphological properties of the lymphatic vessel system in the healed tissues of peri-implant gingival mucosa in humans.
The investigation was performed on fragments of human gingival peri-implant mucosa. Periimplant soft tissue specimens, 2-3 mm wide, were obtained from the peri-implant mucosa during aesthetic gingival remodelling interventions around implant abutments. These samples were obtained from 10 subjects who had undergone implant surgery 18 months before. All the specimens were fixed in an aldehydic mixture in $0.1 \mathrm{M}$ sodium cacodylate buffer ( $\mathrm{pH} 7.4$ ), postfixed in $1 \%$ $\mathrm{OsO}_{4}$ in $0.2 \mathrm{M}$ collidine buffer ( $\mathrm{pH} \mathrm{7.4)}$ ) and then routinely processed for transmission electron microscope (TEM) observation.

In all the samples the gingival mucosa had transformed to peri-implant mucosa following implant treatments. The bucco-lingual sections of the peri-implant mucosae had a multilayered keratinized epithelium on the side of the oral cavity, while thinner epithelium was present in the side facing the abutment. Loose connective tissue with harboured collagen fibres and cells was interposed between the epithelial coatings. The cells were mainly fibroblasts but some inflammatory cells were also present. Deep papillae of connective tissue protruded from the lamina propria towards the oral epithelium while the border between the lamina propria and the sulcular epithelium was more regular and lacked indentations. A well-developed and uniformly arranged 

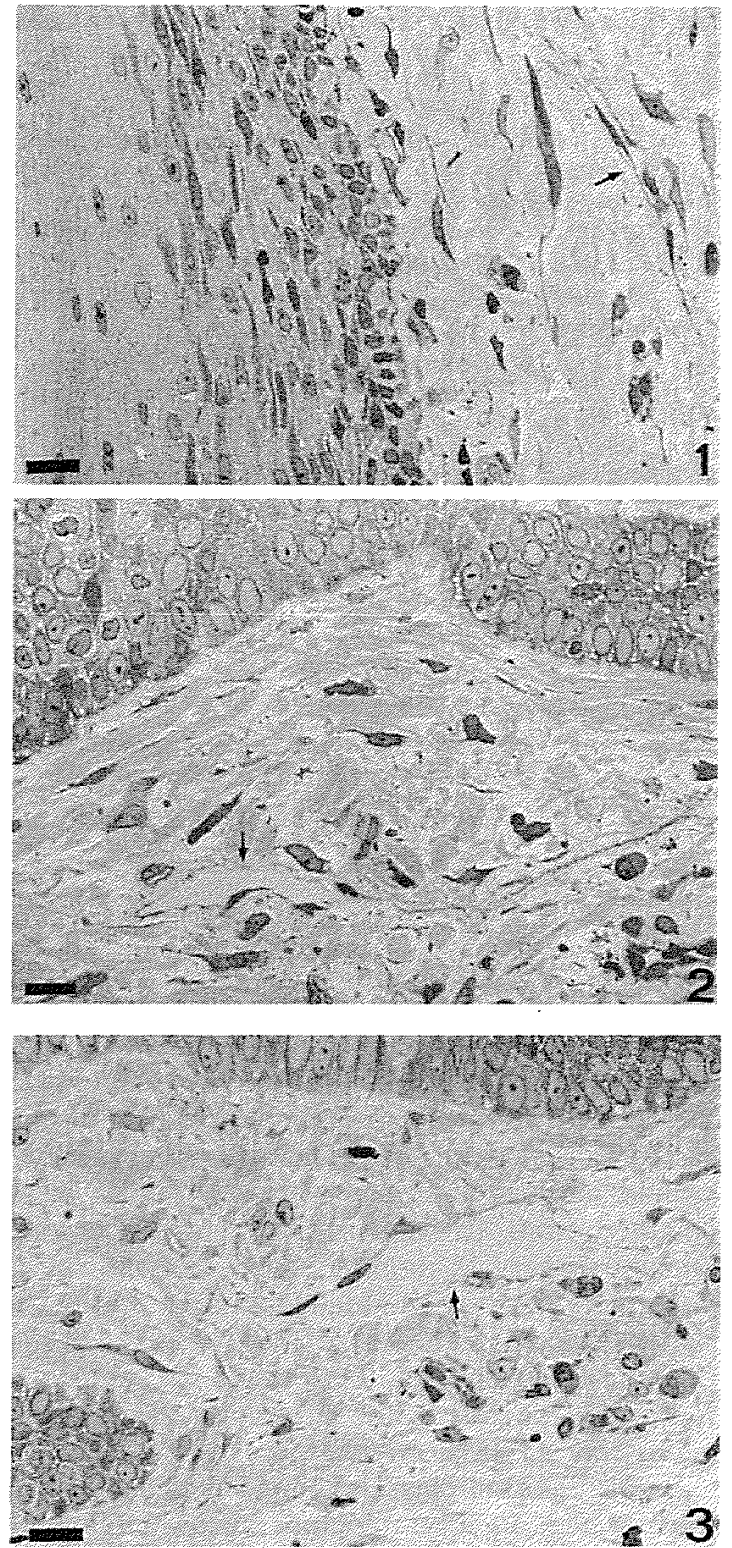

Figs. 1-3. Light micrographs of collapsed (Fig. 1) and variously dilated lymphatic vessels (Figs 2, 3). Magn $400 \times$. Bar $=20 \mu \mathrm{m}$.

network of lymphatic vessels was present in the connective tissue of the lamina propria of all the samples of peri-implant mucosae. The lymphatic vessels were distinguishable from blood capillaries because of their size ( $3-4$ times larger) and from venules because of their shape and the structure of their wall. The lymphatic vessels consisted of thin, irregularly shaped vessels with reduced lumina (Fig. 1) or vessels with more evident lumina (Fig. 2, 3 ) all of which were delimited by a very thin wall.



Fig. 4. Electron micrograph of a very flattened lymphatic capillary with a reduced lumen $\left({ }^{*}\right)$ delimited by a thin and irregular endothelial wall. "Anchoring filaments" (arrows) connect the vascular wall to the collagen fibrils of the connective tissue. Magn $9700 \times$. Bar $=1 \mu \mathrm{m}$.

Figs 5-6. Electron micrographs of sections of lymphatic capillaries with evident lumina. Ovellappings (arrows), intercellular channels (*) and open junctions (arrowed) are present between contiguous endothelial cells. Magn $8400 \times$. Bar $=$ $1 \mu \mathrm{m}$. 
Lymphatic vessels originating in the connective tissue papillae were also seen.

The lymphatic vessels observed by light microscope in the peri-implant tissues had the ultrastructural characteristics of "initial" or capillary vessels (Figs 4-6). Their wall consisted of a flattened endothelium lacking pericytes and a muscular sheath, and was surrounded only by a discontinuous basement membrane and little bundles of "anchoring filaments". These filaments were connected to the collagen fibre network of the tissue (Fig. 4). Overlappings or more intricate junctions between multiple cellular protrusions generally connected the contiguous endothelial cells. On serial sections the cellular edges could be seen to form inter-endothelial channels opening towards the vessel lumen or the surrounding connective tissue matrix (Fig. 5). Intercellular clefts or open junctions (1-2 $\mu \mathrm{m}$ wide) were rarely present in more dilated vessels with a distended wall (Fig. 6).

Our present study has demonstrated for the first time that a network of vessels with the morphological characteristics of lymphatic capillaries also reforms in peri-implant tissues. The lymphatic capillaries of the peri-implant mucosa are distributed in the tissue right up to the junctional epithelium as we had observed previously in healthy and inflamed gingiva (4) and as already demonstrated for the blood vessel plexus (5). Concerning the morphology of the lymphatic vessels, we observed both collapsed and distended vessels, similar to those of the healthy and moderately inflamed gingiva.

From our data we may state some first conclusions:

- In successful implants restored tissue functionality is supported by well-developed networks of both blood and lymphatic vessels.

- No substantial differences exist in localization between the lymphatic vessels in the gingiva and those in peri-implant tissues. The degree of distension of the vessels and the presence of open junctions between contiguous endothelial cells correspond to morphological adaptations of the endothelial cells to different phases of absorbtion by the capillary vessels $(3,4)$.

Our observation that only some lymphatic capillaries present the morphological features of dilated vessels suggests that the lymphatic system is not particularly implied in absorbing activities in normally restored tissue. In other words, the mechanical disruption of the mucosa occurring at the installation of the abutments does not compromise the mucosal barrier function; and the restored epithelium facing the implant surface may maintain the permeability that characterizes the junctional epithelium of the gingiva. Therefore the migration of cells and the drainage of fluids from the connective tissue may be guaranteed by both the junctional epithelium and the lymphatic capillary system in the gingiva as in the periimplant tissues.

\section{References}

1. Ingman $\mathbf{T}$, Kononen $\mathrm{M}$, Konttinen $\mathrm{YT}$, Siirila $\mathrm{HS}$, Soumalainen $K$, Sorsa $T$. Collagenase, gelatinase and elastase activities in sulcular fluid of osseointegrated implants and natural teeth. $J$ Clin Periodontol 1994; 21:301-307.

2. Bernick S, Grant DA. Lymphatic vessels of healthy and inflamed gingiva. $J$ Dent Res 1978;57:810-817.

3. Marchetti C, Poggi P, Icaro Comaglia A, Rizzo S, Rodriguez Y Baena R. Morphological peculiarities of lymphatic capillaries in masticatory and lining mucosa. Lymphology 1998;31:79-82.

4. Marchetti C, Poggi P, Icaro Cornaglia A, Farina A, Rizzo S. Morphologic characteristics of initial lymphatics of the healthy and diseased human gingiva. Anat Rec 1999, in press.

5. Berglundh $T$, Lindhe J, Jonsson $K$, Ericsson I. The topography of the vascular systems in the periodontal and peri-implant tissues in the dog. $J$ Clin Periodontol 1994:21:189-193. 
This document is a scanned copy of a printed document. No warranty is given about the accuracy of the copy. Users should refer to the original published version of the material. 